\title{
Modelling the Formation of Trail Networks by Foraging Ants
}

\author{
James Watmough† AND Leah Edelstein-Keshet $\ddagger$ \\ Department of Mathematics, University of British Columbia, \\ Vancouver, BC V6T 1Z2, Canada
}

(Received on 20 October 1993, Accepted in revised form on 8 May 1995)

\begin{abstract}
This paper studies the role of chemical communication in the formation of trail networks by foraging ants. A cellular automaton model for the motion of the ants is formulated, which assumes that individuals interact according to a simple behavioural algorithm. The ants communicate by depositing trail markers composed of volatile chemicals that serve as attractants for other ants. The ants interact with the network both by following the trails and by extending and reinforcing the trails they follow. By varying the parameters describing these interactions we determine how variations in the behaviour of the individual ants lead to changes in the patterns of trail networks formed by the population. The results indicate that the ability of the group to form trails is inversely related with individual fidelity to trails.
\end{abstract}

(C) 1995 Academic Press Limited

\section{Introduction}

In this paper we develop a cellular automaton (CA) model for the formation of trail network patterns by foraging ants. These patterns are formed by many species of ants during both foraging and migrations (Hölldobler \& Wilson, 1990). As each ant moves it deposits a chemical pheromone which serves as a trail marker and an attractant to other ants. The coupling between the natural decay of the pheromone and the reinforcement of the trails by the attracted ants can lead to the formation of a variety of patterns. Several examples are shown in the sketches in Schneirla (1971), Rettenmeyer (1963) and Raignier \& van Boven (1955). The ability of the ants to forage as a group is essential to the survival of the colony. This implies that the trail patterns must be robust and reproducible in a noisy environment. However, if the society is to be able to adapt to the changing needs of the population, these patterns must also be flexible. We suggest here that both of these requirements are met if the patterns are a consequence of the behaviour of individuals in the population. Over the long term, evolution can lead to

† E-mail: watmough@math.ubc.ca

\#E-mail: keshet@math.ubc.ca changes in individual behaviour that result in different trail patterns; in the short term, patterns can be reproduced by the group under a wide range of conditions.

The goal of this study is to understand how the behaviour of the ants determines the patterns of the resulting trail networks. We therefore base the CA model on several parameters that describe the behaviour of the individual ants, and which can be varied to investigate their effects on the trail network patterns. The parameters characterize the movement of the ants, the exchange between ants that are following trails and ants that are creating new trails, and the stability of the chemicals used as trail markers. This model is based on a previous model by Ermentrout \& Edelstein-Keshet (1993), which was used to study the formation of a trail by ants moving and interacting on a periodic domain. Their results showed that the formation of a dominant trail depends on the total ant density. In this paper, we do not examine the steady-state situation, but concentrate on the initial formation of a trail by ants emerging from a central nest location.

The paper is organized as follows. In Section 2 we develop an algorithm for the motion of each individual 
ant, and describe a simulation based on this algorithm. The results of experimentation with this simulation are presented in Section 3.

\section{Modelling and Simulation}

Cellular automata (CA) simulations are widely used to model pattern formation in the natural sciences. Ermentrout \& Edelstein-Keshet (1993) describe two basic classes of CA simulation: (1) an Eulerian or deterministic model, in which a state value is associated with each point on a spatial grid, and the change in the state with each time step is a deterministic function of the states of the neighbouring grid points; and (2) a Lattice Gas model, in which particles move about and interact on a spatial grid. In the latter case, the motion of the particles has a random component. The spatial and temporal domains are discretized for both methods (see also Schweitzer et al., 1995).

Our model couples a deterministic model for the formation and decay of trails with a lattice gas model for the motion of the ants. The concentration of trail pheromone is represented by a state value of each point in the lattice. Each individual ant is described by a position on the lattice and a velocity. At each time step, the new trail concentration is a deterministic function of its current value (evaporation) and the number of ants at that lattice point (trail deposition). The new position and velocity of each ant are computed using a random walk, biased by the local trail concentration.

The algorithm for the behaviour of each ant is given by the following rules:

1. The ants move at a fixed speed.

2. As each ant moves it deposits a trail pheromone at a constant rate.

3. The trail pheromone evaporates at a steady rate.

4. There is a chance that an ant following a trail will lose the trail. The probability that the ant will remain following a trail per unit time is called the fidelity, and is assumed to be a function of the pheromone concentration.

5. The fidelity is also used to determine if an exploratory ant encountering a trail will start to follow it. If the ant does not follow the trail it will continue to move randomly.

6. When an ant following a trail comes to a fork it will choose one of the branches according to the Fork Algorithm described below.

The first rule is implemented only for convenience. The speed of the ants does in fact vary, but this is not thought to be an essential feature of the problem. The assumption that the pheromone is deposited at a constant rate greatly simplifies the model. Unfortunately there has been very little experimental research into such details. Aron et al. (1989b) note that $I$. humilis foragers make continual contact with the substrate during foraging. This suggests that they may be marking continuously; although there is no direct evidence to confirm this. The observations of Aron et al. (1989b) also suggest that the ants deposit pheromone at different rates if they are travelling towards or away from the nest, and that possibly only a subset of the ants deposit trail markers. These observations have not been incorporated into the model.

To model the limited ability of the ants to follow a trail we assume that there is a non-zero probability that an ant will leave the trail at each time step. This assumption is based on results of experiments indicating that ants follow a trail for only a finite distance (Pasteels et al., 1986; Evershed et al., 1982). These experiments also show that the mean distance an ant follows a trail will increase with the concentration of pheromone on the trail, but that the antennae become saturated at high concentrations of pheromone. This implies that the ant will be able to distinguish between two trails of unequal concentration only if the pheromone concentrations on both trails are below a certain "saturation" level. The ability of an exploratory ant to start following a trail it encounters is modelled using the same parameter. Thus, we assume that there is a non-zero probability that the ant will not turn to follow a trail it encounters. Ants that do not follow the existing trails are considered to be exploring, and move in a random direction. The probability of choosing a given direction depends on the angle of the turn.

There is little information on how an ant will respond to forks in the trail network. We have studied two versions of the implementation of the Fork Algorithm. In the first version, an ant arriving at a trail fork will

(a) continue moving forward if the trail continues straight ahead,

(b) move as if it were exploring if both branches are of equal concentration,

(c) follow the stronger of the two branches if neither of the above conditions are met.

Since the ants are able to detect differences in concentrations between their two antennae, we have assumed that they will turn onto the trail with the higher pheromone concentration. However, we have also assumed that the ants have a strong tendency to continue in their current direction of motion, and will seek out trails to the left and right only if there is no 
trail directly ahead. If the ant encounters two branches of equal strength, both antennae will sense the same pheromone concentration. Since there is no difference in concentration to the left or right, the signal to turn may be absent (Calenbuhr \& Deneubough, 1992; Calenbuhr et al., 1992). In this case, rather than have the ant choose a random branch, we allow it to move as if it were exploring. This random move allows the ant to select one of the existing trail branches in the next time step, and incorporates the characteristics of the exploratory movements of the ants into the decision. This implementation can be expected to produce straighter trails than if one of the existing branches was chosen, since there is a small chance that the exploration will lead away from both trails.

The second version of the Fork Algorithm uses a more flexible approach. The follower chooses a random branch, where the probability of choosing a given branch is proportional to the pheromone concentration on that branch and the angle of the turn. The bias assigned to each angle is a parameter of the simulation. The first version of above algorithm can be considered to have an "extreme forward bias", since the ants will always choose the forward branch if one is available.

The computer simulations were written for the IBM PC, partly in Assembly Language, and partly in C. A rectangular lattice with a maximum size of $256 \times 256$ points was used to represent the spatial domain. The simulation begins by releasing ants, at a rate of one per iteration, from a central nest location. The initial orientation of the ant is chosen randomly from a set of directions specified for each run. At the beginning of each time interval, each ant surveys the immediate neighbourhood and chooses a new direction according to the above algorithm. She will then deposit a pheromone droplet at her current position, and move one step in the new direction. Any ant which moves off the lattice is removed from the simulation. The simulation then continues with the calculations for the next time interval.

The absorbing boundaries (the removal of the ants from the edge of the lattice) are an approximation of an infinite domain. The run times for the simulation are kept an order of magnitude below the mean time that an ant would arrive at the boundary by random motion alone. Thus the majority of the ants at the boundaries are on trails leading out of the region. Further, we are concerned with the initial formation of the foraging trails, and can therefore assume that the return traffic is negligible. (See the description of the natural situation for army ants given in Schneirla, 1971: pp. 89-92).
The following variables and parameters are used:

$$
\begin{aligned}
C(x, t)= & \text { concentration of pheromone at point } x \text { and } \\
& \text { time } t, \\
F(x, t)= & \text { number of trail followers at point } x \text { and time } \\
& t, \\
L(x, t)= & \text { number of exploratory (lost) ants at point } x \\
& \text { and time } t, \\
\tau= & \text { rate of pheromone deposition per ant per } \\
& \text { time step, } \\
\phi(C)= & \text { fidelity or probability per iteration of an ant } \\
& \text { remaining on a trail with a local concen- } \\
& \text { tration of } C, \\
\phi_{\text {low }}= & \text { minimum value of } \phi, \\
C_{s}= & \text { pheromone level at which antennae saturate, } \\
\Delta \phi= & \text { rise in } \phi \text { as } C \text { increases to } C_{s}, \\
\bar{r}= & \text { the mean distance that an ant will follow a } \\
& \text { trail, } \\
B_{n}= & \text { the probability of an exploratory ant turning } \\
& \text { an angle of } 45 n^{\circ} .
\end{aligned}
$$

The fidelity, $\phi$, of the ants to the trails is a function of the trail concentration. For simplicity, we assume the relationship shown in Fig. 1; the fidelity is directly proportional to the trail concentration up to a saturation point, after which it remains constant. $\Delta \phi$ measures the rate of increase in the fidelity with increased pheromone concentration. If the ants have a higher fidelity to the trails, they will tend to remain on a trail longer. The velocity of the ants is assumed to be one lattice point per iteration; therefore the mean distance an ant will follow a trail is given by $\bar{r}=256 /(256-\phi)$ lattice points. (The number 256 is used since the random numbers generated by the computer were between 0 and 256). Although the experiments of Evershed et al. (1982) show an exponential relationship between $\bar{r}$ and the trail concentration, we assume simply that this mean distance is an increasing function of $C$. A related measurable parameter is the rate at which followers lose the trail per unit time $(v / \bar{r}$, where $v$ is the velocity of the ants). This parameter is used in the models of

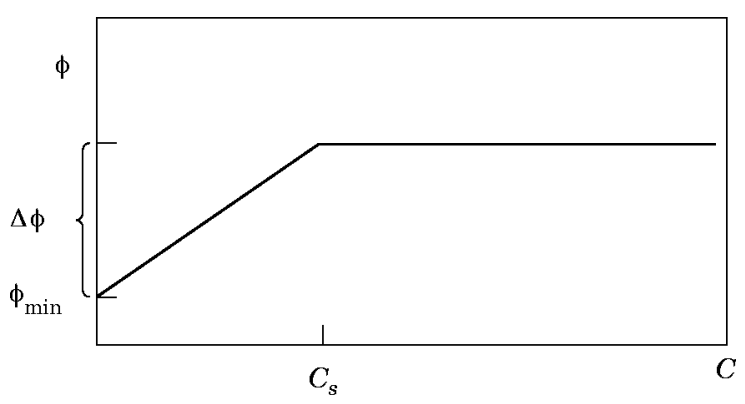

FIG. 1. Graph of $\phi(C)$. The fidelity $\phi$ increases in direct proportion to the trail concentration $C$ up to the saturation point $C_{s}$. 
(a)

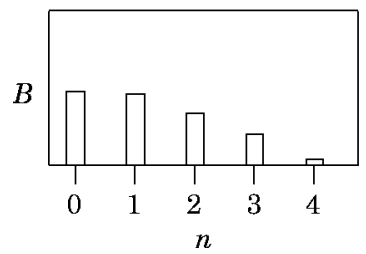

(b)

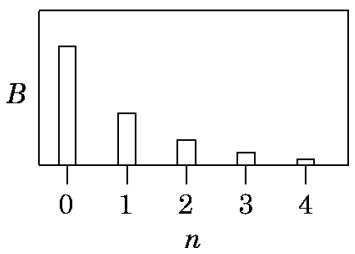

FIG. 2. Two possible turning kernels. Kernel (a) yields wider turns than kernel (b). We can thus distinguish qualitatively between a tendency for wide or narrow turns.

Edelstein-Keshet (1994) and Watmough \& EdelsteinKeshet (1995).

The trail deposition rate, $\tau$, is assumed to be the same for ants following trails as for the exploratory ants. The length of a trail left behind a single individual is directly proportional to $\tau$ (Bossert \& Wilson, 1963). To simplify the calculations we have assumed that the pheromone evaporates at a steady rate. While this yields the correct length of a trail behind a single individual, it predicts a linear decrease in the pheromone concentration along the trail, rather than the inverse relation deduced by Bossert \& Wilson (1963). The overestimate is believed to be acceptable for the model; the important feature is the feedback resulting from the reinforcement of the trails by the followers.

The turning kernel, $B$, characterizes the random turns of the exploratory ants. The lattice used in the simulation allows eight turning directions. If we assume that the probabilities of turning left or right are equal, then the turns can be characterized by the probability distribution $B=\left(B_{1}, B_{2}, B_{3}, B_{4}\right)$, where $B_{n}$ is the probability of turning $45^{\circ} n$ increments either left or right. Figure 2 shows two possible turning kernels. If small angle turns are more common than large turns then a narrow (peaked) kernel similar to that of Fig. 2(b) will result. The wide kernel of Fig. 2(a) indicates a greater chance of larger angle turns (beyond $45^{\circ}$ ).

\section{Results}

Several separate runs of the simulation were performed for various values of the parameters. Our main objective was to study trail morphology and its dependence on the parameters.

The main quantifiers of the trail networks are the strength of the trails and the total length of all trails in a given area. The strength of a trail is defined to be the pheromone concentration per unit length of the trail (Edelstein-Keshet, 1994), and can be related to the density of followers on the trail (followers per unit length of trail). If the majority of the ants are exploratory, then the average strength of the trails will

be proportional to the deposition rate $\tau$. To see this, recall that the ants move at a speed of one step per unit time, and the pheromone evaporates at a rate of one unit of pheromone per unit time. Therefore a deposition rate of $\tau$ units of pheromone per unit time leads to a trail of length $\tau$ behind a single exploratory ant. The concentration of pheromone will decrease linearly with distance along the trail, and so the total pheromone concentration of a simple trail left behind a single exploratory ant will be $\tau+(\tau-1)+\cdots+2+1=\tau(\tau+1) / 2 . \quad$ The average strength (total concentration divided by trail length) of the trail is $(\tau+1) / 2$. If, on the other hand, there is a high density of followers on a single trail then its strength will be significantly increased.

Another quantitative measure of trail strength is the ratio of followers to lost ants. Since all trails are headed by "lost" ants, the ratio is an indication of the average number of followers per trail. Unfortunately, the ratio $F / L$ represents the average trail strength. It does not distinguish between the case where there are several trails of moderate strength, and the case where there are a few strong trails and many weaker trails. This distinction must be made using a visual count of the number of trails.

\subsection{CASE I: CONSTANT FIDELITY $(\Delta \phi=0)$}

In the initial investigations, the parameter $\Delta \phi$ was set to zero. That is, the fidelity was assumed to be independent of the local trail concentration $C$. The results of these simulations were as follows:

\section{The fidelity, $\phi$}

(1) Decreasing fidelity decreases the mean strength of the trails. The ratio $(F / L)$, which is a measure of trail strength, drops from $(F / L)=14$ in Fig. 3(a) to $(F / L)=3$ in Fig. 3(c). This indicates that, as expected, a larger proportion of the population remains on the trails for larger values of the fidelity $\phi$. Note that with $\phi=247, \bar{r}$, the mean length of trail followed by an individual ant is approximately 28 , and with $\phi=254$, $\bar{r} \approx 128$. Hence, the relatively narrow range of $\phi$ used in the simulations corresponds to a wide range of values of the mean length of trail followed $(\bar{r})$. As the fidelity was reduced further, a strong trail failed to form. Results (not shown in figures) for values of $\phi$ below $210(\bar{r} \approx 5)$ show that the network is reduced to many isolated weak trails with very few followers.

(2) The ability of the group to form a dominant trail is inversely related to individual fidelity. Figures 3(a)-(c) show that as $\phi$ is decreased there is a gradual shift from a network with many weak trails and several tortuous, strong trails to a network with several weak trails and a few strong trails. Surprisingly, it appears that the fact 

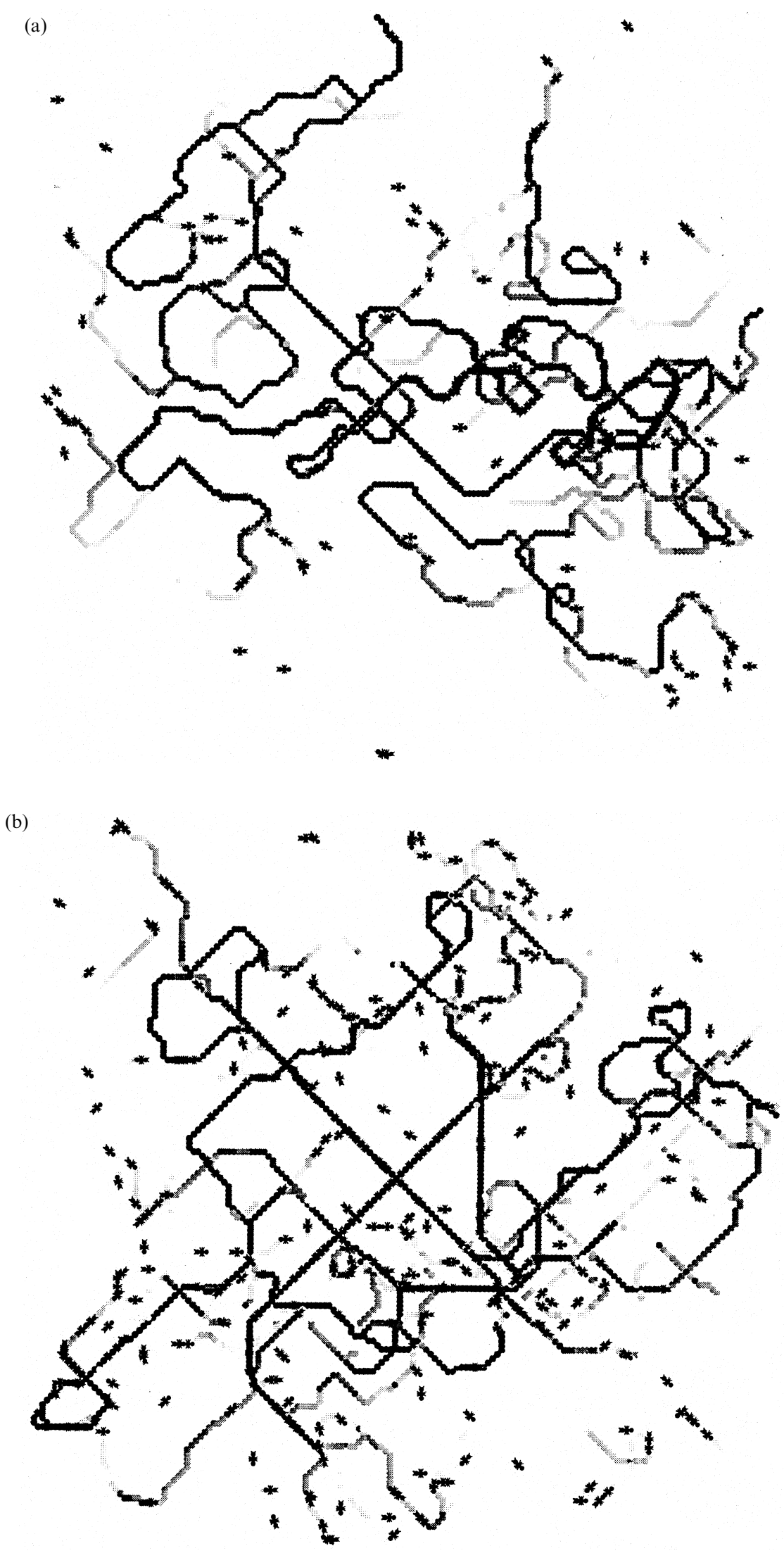

FIG. 3 (a and b). 


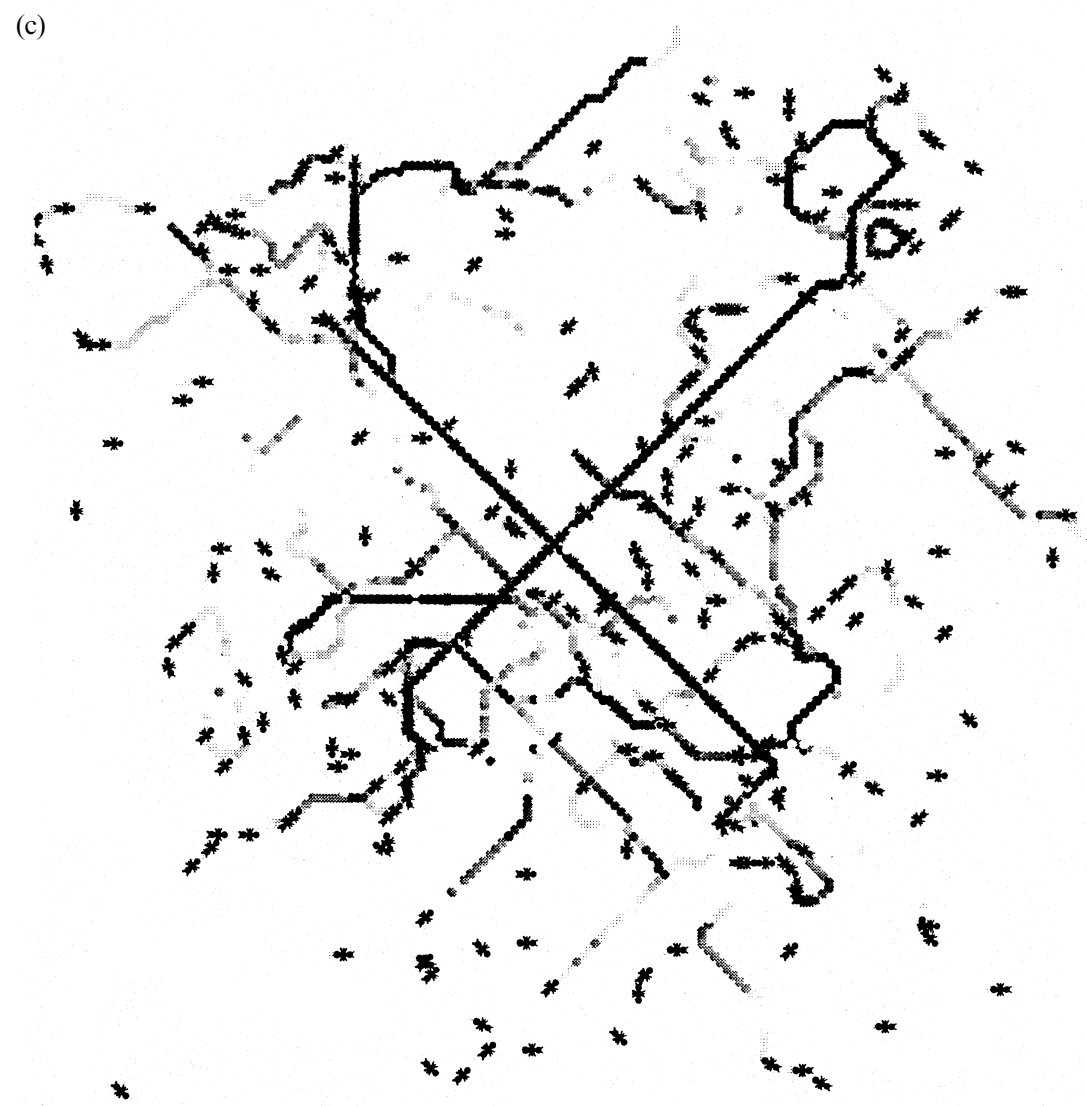

Fig. 3. Three runs summarizing the dependence of trail network on the fidelity of the ants to the trails. In (a) $\phi=255, F=468, L=32$; in (b) $\phi=251, F=396, L=89$; in (c) $\phi=247, F=297, L=91$. As $\phi$ is reduced, the average number of followers per trail decreases; however, this results in a smaller number of strong trails. For each figure $B=(0.360,0.047,0.008,0.004), \tau=8$ and the time is 1500 time steps. In these figures, and in those that follow, the greyscale is a linear function of the pheromone concentration $C$ at each point, with the darker shade indicating a higher concentration. Due to the scale chosen, the weakest trails (behind solitary foragers) appear white. Thus the figures only show trails that have been reinforced by followers.

that the ants are not perfectly dedicated followers and occasionally lose the trail allows them to find and follow stronger trails. This allows the selection of a small number of strong trails, rather than the large number of trails which results from the more dedicated followers. Thus the decrease in the average strength of the trails indicated by the ratio $F / L$ represents a decrease in the number of strong trails, and not a decrease in the strength of the strongest trails.

\section{The deposition rate, $\tau$}

A decrease in the deposition rate leads to a more rapid formation of stronger trails. Figures 4(a)-(c) summarize the effect of changing $\tau$. At a high deposition rate $(\tau=12)$, the network consists of a few strong trails, and many moderate and low strength trails [Fig. 4(a)]. As the deposition rate is decreased, these weaker trails are gradually reduced to solitary lost ants [Fig. 4(b)]. At a low deposition rate $(\tau=4)$, the trail network has only two strong trails and many weak trails consisting of an exploratory ant with no followers [Fig. 4(c)]. In addition, the numbers of exploratory ants and followers are almost equal. Note that although there are fewer followers and the ratio $F / L$ has decreased, this is due to the disappearance of the weaker trails. The dominant trails of Fig. 4(c) are in fact stronger.

The results in Fig. 4 were taken after 4000 iterations. Comparing Fig. 4(b) with Fig. 3(c), which has the same parameter values, shows that the stronger "trunk" trails become more apparent as the network evolves. Thus an increase of $\tau$, which can be thought of as an increase in the influence of each individual, delays the formation of the stronger trails observed in Figs 4(b) and (c).

\section{The turning kernel, $B$}

The turning kernel affects the tortuosity of the trails, and the fraction of the lattice explored by the ants. Figure 5 shows the results of three runs of 1500 iterations in which the kernel is weighted towards narrow turning angles. There are few trails in the network, and a only small fraction of the lattice has 

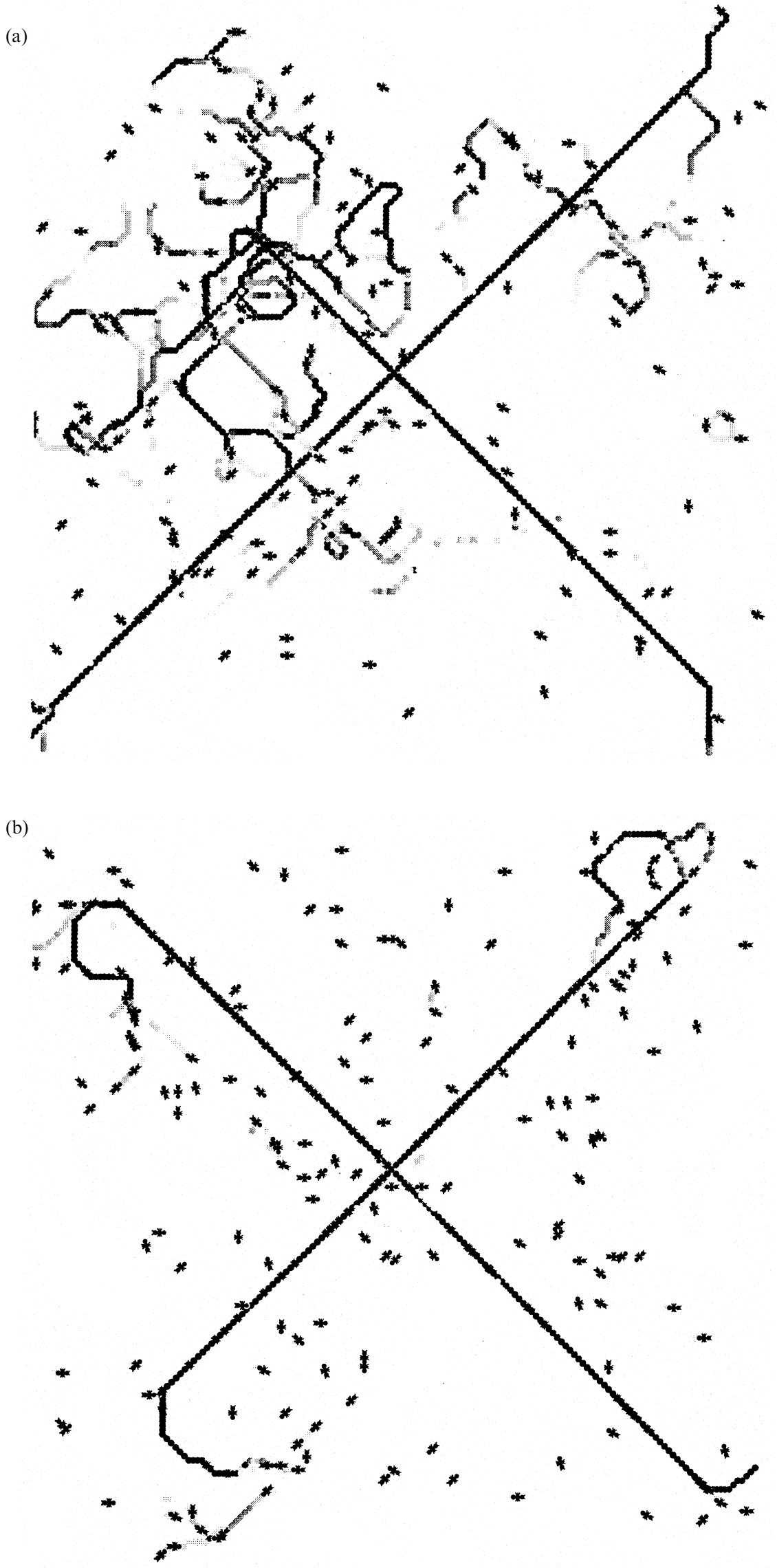

FIG. 4 (a and b). 


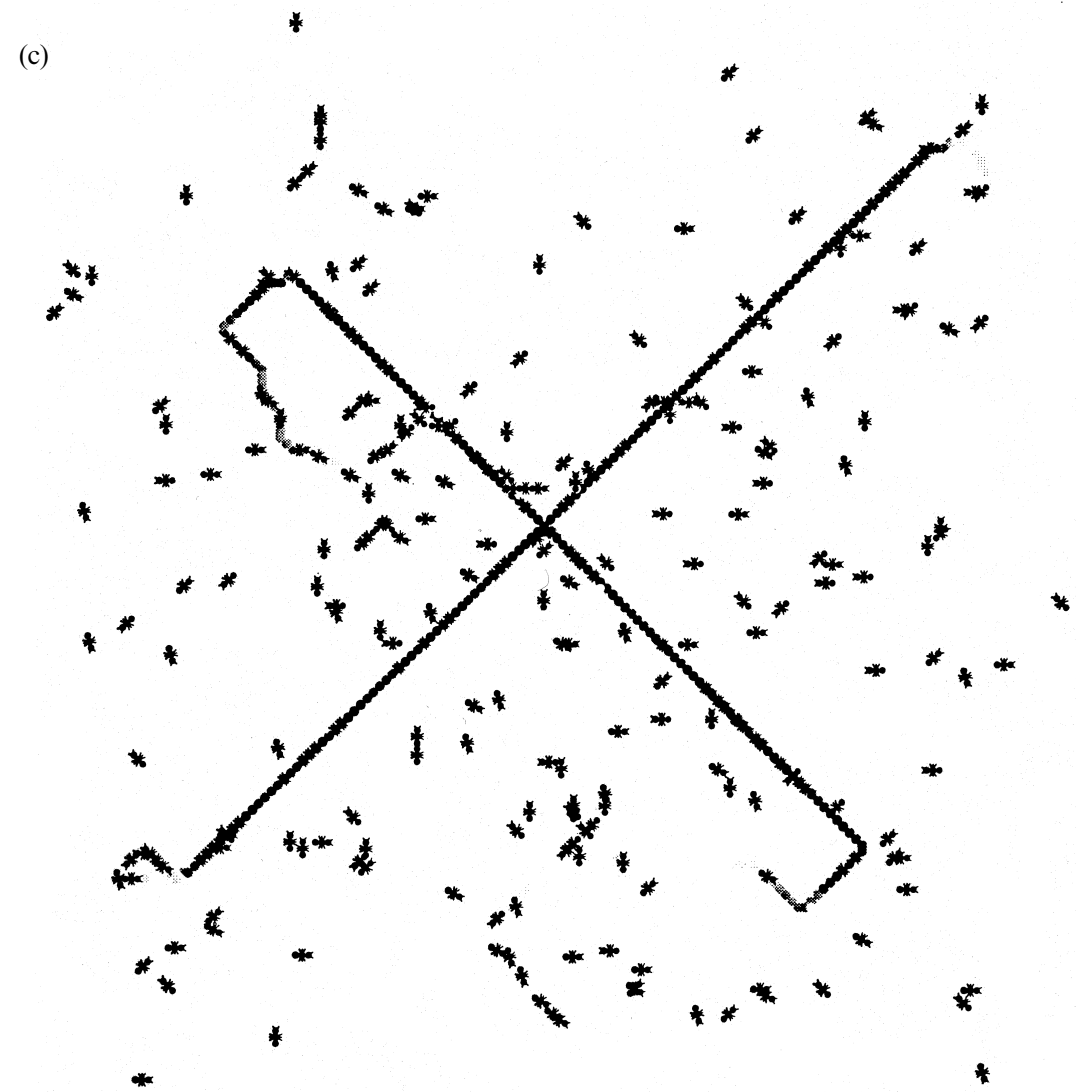

Fig. 4. Three runs showing the dependence of the trail network on the rate of trail deposition. In (a) $\tau=12, F=206, L=77$; in (b) $\tau=8$, $F=148, L=108$; in (c) $\tau=4, F=166, L=167$. As $\tau$ is decreased, the ratio of followers to exploratory ants also decreases, indicating that the average trail strength is lower for smaller values of $\tau$. However, this decrease in average trail strength is due to a decrease in the strength of the weaker trails only. The strength of the stronger trails appears to increase. For each figure $B=(0.360,0.047,0.008,0.004), \phi=247$, and the time is roughly 4000 steps.

been explored. The use of a broader turning kernel, as shown in Fig. 3, results in networks with more trails and a more thorough search of the lattice. Mathematical analysis (Othmer et al., 1988) reveals that in the absence of chemical communication, the more directed kernel leads to a faster spread of the population. The results of the simulation demonstrate that although the trail network is spread over a larger area, the fraction of the area explored decreases as the turn angle distribution narrows.

Figures 3(c) and 4(b) use the same parameter values, but are shown at different times. Figure 5(c) uses the same fidelity and deposition rate, but a narrower turning kernel. For the case of the narrow kernel [Fig. 5(c)], a dominant trunk trail formed after only 1500 iterations, whereas for the wider kernel [Fig. 3(c) and Fig. 4(b)] 4000 time steps were required. The wider kernel leads to the formation of tortuous trails, which take longer to straighten and develop into strong trails.

\section{The fork algorithm}

The above results were obtained using the first version of the Fork Algorithm. A small number of preliminary experiments have been made with the second version of the Fork Algorithm. For this case, the choice of which branch to follow at a trail fork is random. However, there is a higher probability of choosing the stronger branch. In most cases, the results of the simulation did not appear to depend strongly on the version of the Fork Algorithm. The strength of the trails was found to decrease with decreasing fidelity in both versions. Changes in the deposition rate also produced similar results for both versions. Figure 7 shows the results of a simulation using a uniform follower kernel. Lowering the deposition rate leads to a single dominant "trunk" trail. In both runs, the value of the fidelity was only 239 . Similar results were obtained for higher values of the fidelity. The only difference noted between the two versions was that the number of strong trails in the network did not increase with decreasing fidelity using the second version of the algorithm.

\subsection{CASE II: INCREASING FIDELITY TO STRONGER TRAILS}

$$
(\Delta \phi \neq 0)
$$

There is evidence (at least for some ant species) that the fidelity $\phi$ is an increasing function of the trail 

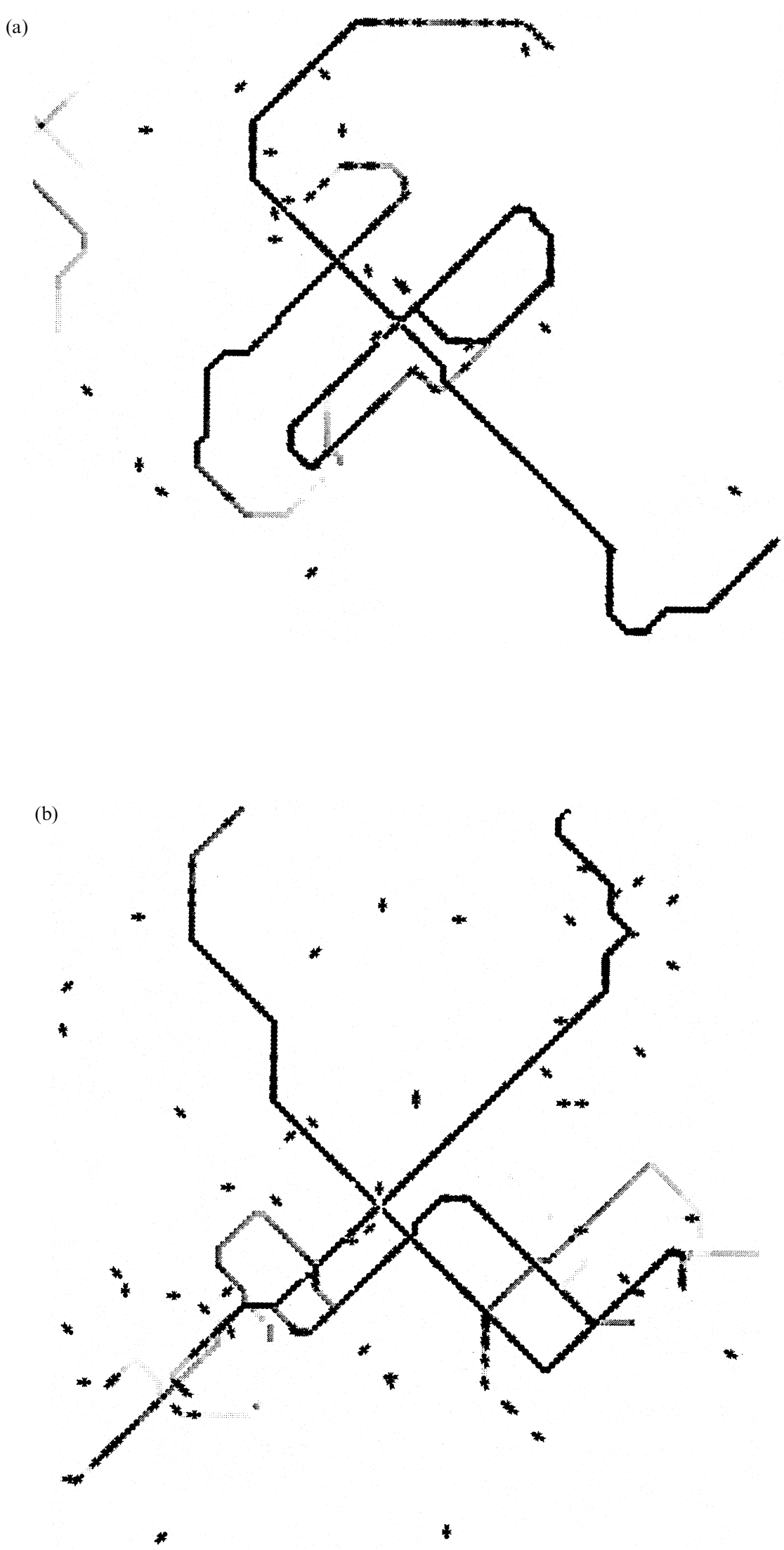

FIG. 5 ( $a$ and b). 


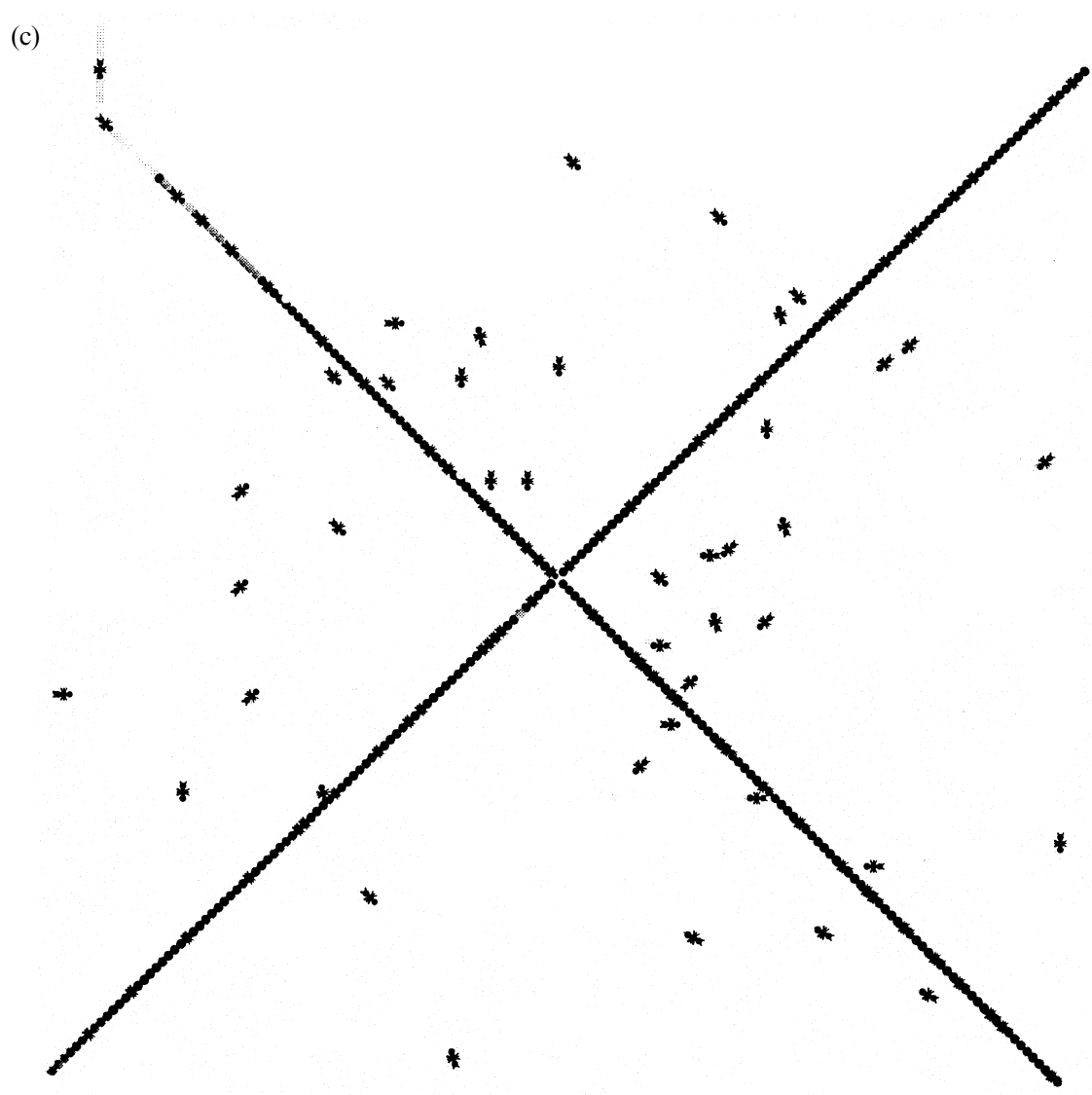

FIG. 5. These three runs were performed with a turning kernel which favoured narrower turn angles and should be compared with the runs of Fig. 3, which showed the same progression of decreasing trail fidelity but favoured wider turns. With the narrow kernel, the number of trails and the proportion of the lattice explored by the ants have both reduced. That is, although the networks are spread over roughly the same area, those of Fig. 5 cover a smaller portion of that area than those of Fig. 3. In (a) $\phi=255, F=141, L=16$; in (b) $\phi=251, F=129$, $L=47$; in (c) $\phi=247, F=27, L=41$. For each run, $B=(0.135,0.031,0.008,0.004), \tau=8$, and the time is roughly 1500 steps.

strength (Pasteels et al., 1986; Evershed et al., 1982). Several runs of the simulation were performed with $\phi_{\text {low }}=20$ and $\Delta \phi=235$. By varying the pheromone level at which the antennae saturate, $C_{s}$, we study the evolution of the trail network as a function of the rate of increase of the trail fidelity, with the trail concentration $\left(\Delta \phi / C_{s}\right)$. As $C_{s}$ was increased, fewer strong trails were formed. Figure 6(b) shows that a pair of strong trails has developed after 500 iterations. In Fig. 6(a), there are still several strong trails competing for dominance after the same number of iterations.

\section{Discussion}

The results of the simulation show that a network of trails can form for any set of parameter values; however, the network gradually loses its structure as the fidelity of the ants to the trails $(\phi)$ is decreased. This is an indication that the formation of the trail networks is a result of the feedback between the natural decay of the trails and their selective reinforcement by the followers. The addition of a single follower to a trail increases the lifetime of that trail. This, in turn, increases the probability that more exploratory ants will find and follow that trail before it evaporates. In a simple situation with only two trails, such as the experimental setup of Beckers et al. (1992), any difference in length of the two trails will be magnified by this feedback process.

The formation of stronger trails also depends on the pheromone deposition rate, $\tau$. For high values of the deposition rate, each ant lays a relatively stronger trail. If the deposition rate is lowered, these individual trails become less effective at attracting new recruits and begin to fade faster than they are reinforced. This means that there will be fewer contradictory signals to divert traffic, and strong trails, once formed, will dominate.

By varying individual parameters, we observe a switch in the resulting pattern from trails which have very few followers to trails which may be of comparable length but are more heavily marked and 
(a)

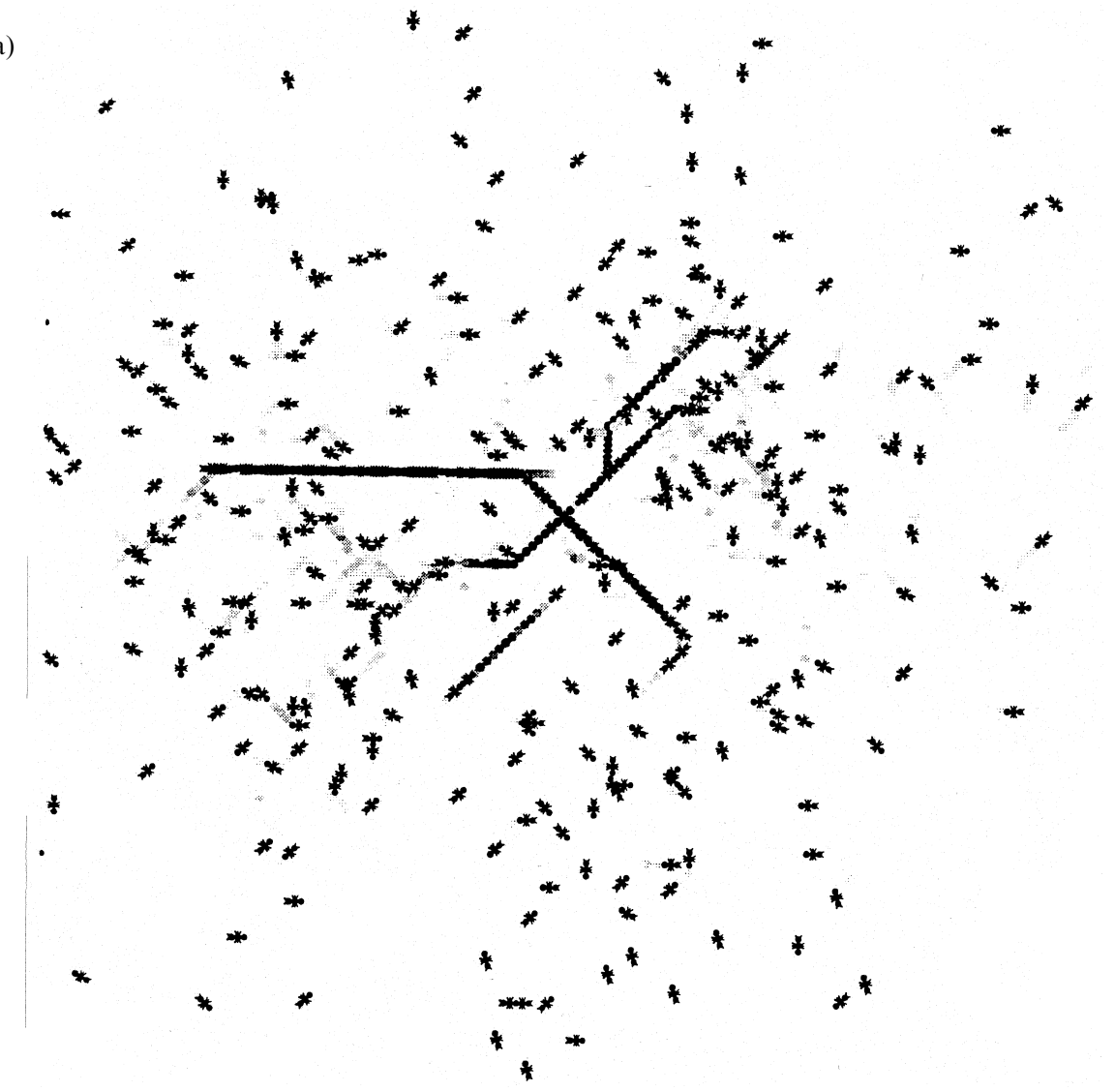

(b)

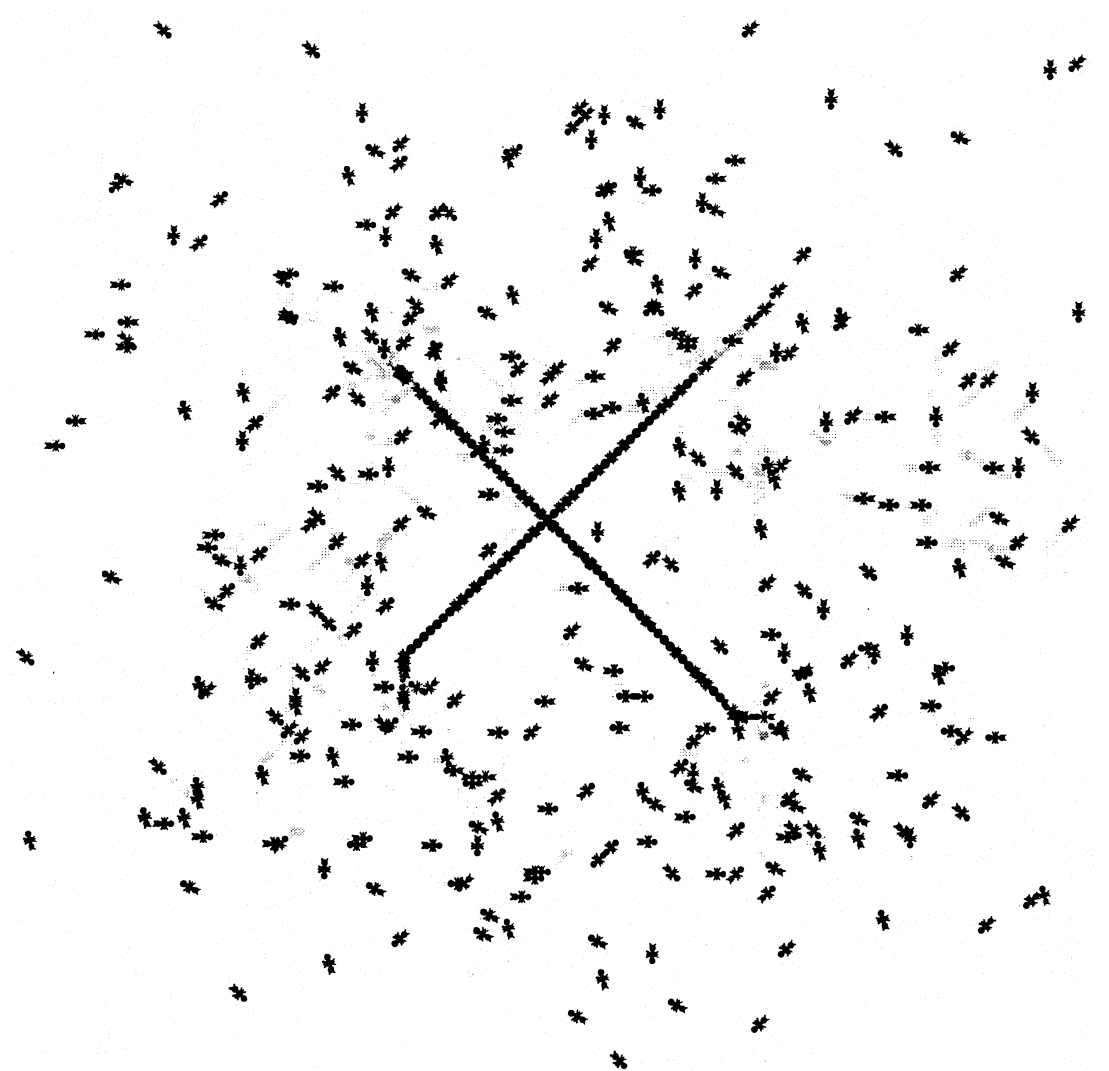

FIG. 6. Two runs in which the fidelity $(\phi)$ was an increasing function of the trail strength as defined in the text. (a) $C_{s}=6, F=145, L=207$; in (b) $C_{s}=18, F=89, L=299$. In each run $B=(0.360,0.047,0.008,0.004), \phi_{\text {low }}=20, \Delta \phi=235$ and $\tau=6$. As $C_{s}$, the level at which the antennae saturate, increased, the number of strong trails decreased. 
carry a higher proportion of traffic. Changes in parameters can also result in a change in the number of trails in the network as well as the tortuosity of the trails. Such transitions are also observed in the patterns formed by army ants. E. burchelli will, over a few hours, switch from the broad, area covering fan used for foraging, to a single trunk trail used for migrations. Our results suggest that this switch in group behaviour could arise from a small shift in individual behaviour. Such a shift could be triggered by the release of primer pheromone or some other signal.

Over a longer, evolutionary time scale, our model suggests that variations in the raiding patterns between species can be accounted for by variations in individual behaviour. These variations may be due to differences in the preferred prey of the species. Several previous simulations and experiments have shown that a change in the food source can lead to a change in the pattern of group foraging (Deneubourg et al., 1989, Franks, 1993). These models attribute the formation of trail networks to a difference in pheromone deposition rates between ants returning to the nest with food and foragers without food. Our model shows that the different patterns may also occur without this distinction. For species whose prey has a homogeneous distribution, such as E. burchelli, it would be advantageous for each ant to have a higher affinity to trails or a higher pheromone deposition rate than a species such as $E$. hamatum, which feeds on a patchy distribution of food. The lower affinity to trails would allow E. hamatum to quickly develop a strong trail to the best food supply. Note that an increase in the

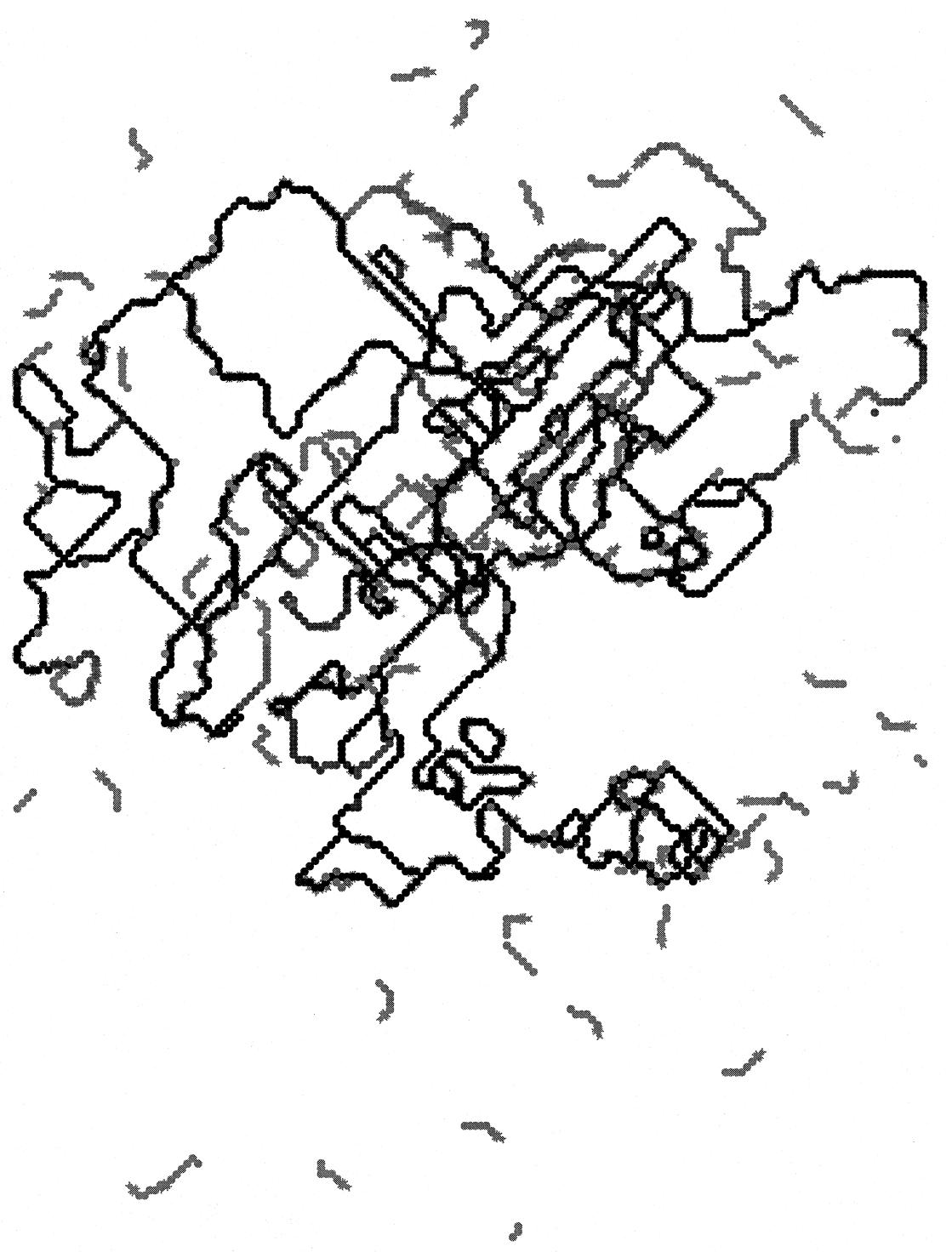

FIG. 7 (a). 


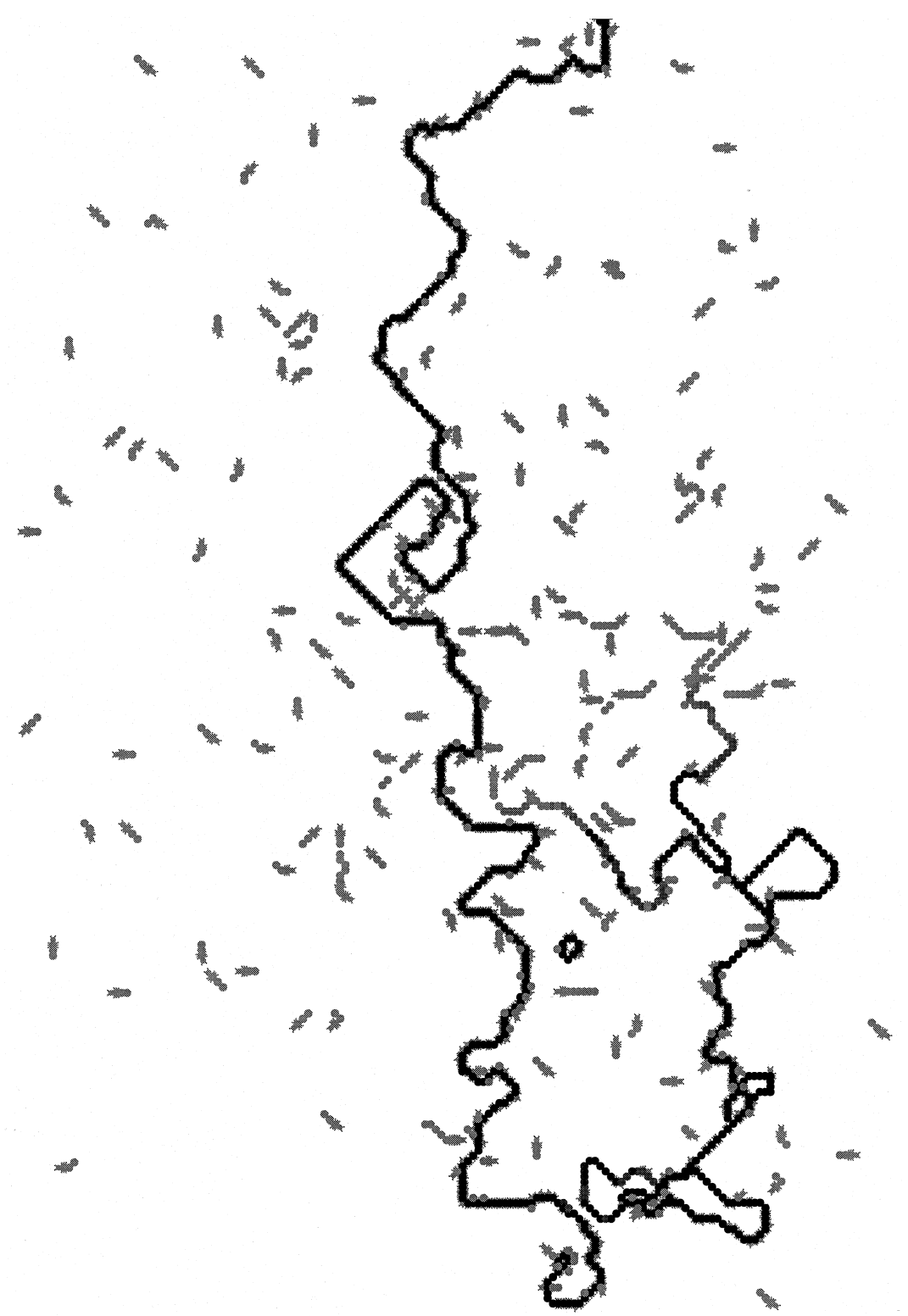

FIG. 7. Two runs using a uniform follower kernel. In (a), $\tau=8, \phi=239, F=845, L=105$. In (b) $\tau=4, \phi=239, F=788, L=212$. A dominant "trunk" trail will form for lower values of the deposition rate $\tau$. For both runs $B=(0.360,0.047,0.008,0.004)$ and the time is 2000 steps.

amount of pheromone deposited per individual is not required for the formation of these more directed networks. In fact, our model predicts that the formation of a dominant trunk trail would be postponed if $\tau$, the deposition rate, were increased, since this tends to promote the formation of many competing trails. The greater strength of the trunk trail is achieved by the collective effort of many ants laying weak trails. A similar result was obtained in the experiments of Beckers et al. (1990), which compared the ability of the trail recruiters L. niger and the group recruiters $T$. caespitum to discover and exploit food sources. If one considers group recruitment to be similar to a very large affinity to existing trails, then the experiment shows that the limited ability of $L$. niger to follow trails allows it to discover and exploit new food sources even after a strong trail to an existing food source has developed. Conversely, the superior ability of $T$. caespitum individuals to follow (recruit to) an existing, strong trail lowers the colony's ability to exploit a second food source. Millonas (1992) has developed a model to examine the choice of branches at trail forks. His results also show this inverse relationship between individual and group ability.

The parameters used in the model can be determined experimentally. This has been done for several species 
(see summary in Edelstein-Keshet et al. 1995). Unfortunately, there is a great variation from species to species, and there does not appear to be a complete set of parameter values measured for a single species. We can estimate some of these parameters to gain an understanding of the scale of our simulations. The speed of E. hamatum along trails is given as $4 \mathrm{~cm} \mathrm{~s}^{-1}$ (Schneirla, 1971). If we assume that each iteration is one second then the grid spacing will be $4 \mathrm{~cm}$. A deposition rate $\tau=7$ leads to a trails of $28 \mathrm{~cm}$ behind a single exploratory ant, which is the value given by Bossert \& Wilson (1963). The time required for the formation of a dominant trail is given as $30 \mathrm{~min}$ (Schneirla, 1971), which is equivalent to 1800 iterations (seconds). Again, these are of the same order as those predicted by the simulations. A rather unfortunate consequence of our desire to track the motion of each individual ant is that the computation times increase geometrically with the number of ants. While the simulations were performed in a reasonable time for small numbers of ants, the number of ants in typical colonies is at least three orders of magnitude higher. With such large population densities, it is no longer realistic to use these models; however, the examination of these mechanics at low densities does lead to a greater understanding of the problem.

The algorithm used for the motion of the ants in this paper has also been used to derive a system of partial differential-integral equations (PDIEs) which describe the motion of the swarm and the evolution of the trail network. While these equations allow us to study the consequences of larger densities, they unfortunately prove difficult to analyse. A separate paper (Watmough \& Edelstein-Keshet, 1995) deals with the mathematical analysis of a simpler one-dimensional version of the model. Results of a space-independent model by Edelstein-Keshet (1994) indicate that the dependence of the fidelity on trail concentration is important in the creation of a network of strong trails rather than a network of weak trails. Experimental evidence suggests that the mean length of a trail followed by an ant will increase with the strength of the trail (Pasteels et al., 1986; Evershed et al., 1982). Experiments with our CA model also indicate that such a dependence leads to a faster emergence of stronger trails in the network.

The use of the CA simulations allows us to explore the development of the network in two dimensions without making many simplifying assumptions, as might be required in the case of a mathematical analysis. Further, the results of the simulation can be displayed in "real time" on the computer screen. These animated visual images provide an excellent means to study the problem and to display the results. The insight provided by this experimentation can be used to direct a further, more detailed mathematical analysis of the problem. Such analysis is still necessary as it is not possible, using the simulation, to place definite bounds on regions of parameter space in which different results occur.

Hidden in our thesis is the assumption that the trail networks created by the ants are self-organized (Pasteels et al., 1987; Aron et al., 1989a; Deneubourg \& Goss, 1989; Beckers et al., 1990; Deneubourg et al., 1990, 1991). This indicates that the patterns emerging at the level of the group are the result of interactions between many similar individuals; each individual performs a simple task, and none acts as a leader or organizer. Our model allows us to determine how the different aspects of the behaviour of the individuals affect the trail patterns that emerge from the group motion. By varying these parameters individually, we are able to determine their role in the development of the trail network. If communities of social insects are self-organized, then studies such as these are a first step in understanding how such communities arise and evolve. We have shown that only minor changes in the behaviour of the individuals are necessary to enable the society to adapt to much larger changes in the environment.

We gratefully acknowledge support from the National Sciences and Engineering Research Council of Canada under grant number OGPIN 021. We also wish to thank Bard Ermentrout and Scott Camazine for helpful comments and discussions of this model.

\section{REFERENCES}

Aron, S., Deneubourg, J. L., Goss, S. \& Pasteels, J. M. (1989a). Functional self-organization illustrated by inter-nest traffic in ants: the case of the argentine ant. In: Biological Motion, Proceedings, Königswinter. Lecture Notes in Biomathematics, Vol. 89 (Alt, W. \& Hoffmann, G., eds) pp. 533-547 Berlin: Springer Verlag.

Aron, S., Pasteels, J. M. \& Denoubourg, J. L. (1989b). Trail-laying behaviour during exploratory recruitment in the argentine ant, Iridomyrmex humilis (Mayr). Biol. Behav. 14(3), 207-217.

Beckers, R., Deneubourg, J. L., Goss, S. \& Pasteels, J. M. (1990). Collective decision making through food recruitment. Insectes Sociaux (Paris), 37(3), 258-267.

Beckers, R., Deneubourg, J. L. \& Goss, S. (1992). Trails and U-turns in the selection of a path by the ant Lasius niger. J. theor. Biol. 159, 397-415.

Bossert, W. \& Wilson, E. O. (1963). The analysis of olfactory communication among animals. J. theor. Biol. 5, 443-469.

Calenbuhr, V. \& Deneubourg, J. L. (1992). A model for osmotropotactic orientation (I) J. theor. Biol. 158, 359-407.

Calenbuhr, V., Crétien, L., Deneubourg, J. L. \& Detrain, C. (1992) A model for osmotropotactic orientation (II). J. theor. Biol. 158, 359-407.

Deneubourg, J. L. \& Goss, S. (1989). Collective patterns and decision making. Ethol. Ecol. Evol. 1, 295-311.

Deneubourg, J. L., Goss, S., Franks, N. R. \& Pasteels, J. M. (1989). The blind leading the blind: Chemically mediated morphogenesis and army ant raiding patterns. J. Insect Behav. 2, 719-725. 
Deneubourg, J. L., Aron, S., Goss, S. \& Pasteels, J. M. (1990). The self-organizing exploratory pattern of the Argentine ant. J. Insect Behav. 3(2), 159-168.

Deneubourg, J. L., Goss, S., Beckers, R. \& Sandini (1991) Collectively self-solving problems. In: Self organization, Emergent Properties, and Learning. (Babloyantz, A. ed.) New York: Plenum.

EDELSTEIN-KeSHET, L. (1994). Simple models for trail-following behaviour: Trunk trails versus individual foragers. J. math. Biol. 32, 303-328.

Edelstein-Keshet, L., Watmough, J. \& Ermentrout, G. B. (1995). Trail-following in ants: Individual properties determine population behaviour. Behav. Ecol. Sociobiol. 36, 119-133.

Ermentrout, B. \& Edelstein-Keshet, L. (1993). Cellular automata approaches to biological modeling. J. theor. Biol. 160 , 97-133.

Evershed, R. P., Morgan, E. D. \& Cammaerts, M. C. (1982). 3-ethyl-2,5-dimethylpyrazine, the trail pheromone from the venom gland of eight species of Myrmica ants. Insect Biochem. 12(4), 383-391.

Franks, N. (1993). The blind leading the blind: Chemically mediated morphogenesis and army ant raid patterns. J. Insect Behav.

Hölldobler, B. \& Wilson, E. O. (1990). The Ants. Cambridge, MA: Harvard University Press.

Millonas, M. M. (1992). A connectionist type model of self-organized foraging and emergent behaviour in ant swarms J. theor. Biol. 159, 529-552.

Othmer, H. G., Dunbar, S. \& Alt, W. (1988). Models of dispersal in biological systems. J. math. Biol. 26, 263-298.

Pasteels, J. M., Deneubourg, J. L., Verhaeghe, J. C. \& Boevé, J. L. (1986). Orientation along terrestrial trails by ants. In: Mechanisms in Insect Olfaction (Payne, T. L., Birch, M. C. \& Kennedy, C. E. J., eds) pp. 131-138. Oxford: Clarendon Press.

Pasteels, J. M., Deneubourg, J. L. \& Goss, S. (1987). Self-organization mechanisms in ant societies (I): Trail recruitment to newly discovered food sources. In: From Individual to Collective Behavior in Social Insects pp. 155-175. Basel: Birhauser.

Raignier, A. \& VAN Boven, J. K. A. (Pasteels, J. M. \& Deneubourg, J. L., eds) (1955). Etudé taxonomique, biologique et biométrique des dorylus du sous-genre anomma (Hymenoptera: Formicidae). J. Annal. Musée roy. Congo Belge ns 4 sci. zool. 2, $1-359$.

RetTenmeyer, C. W. (1963). Behavioral studies of army ants. Univ. Kansas Sci. Bull. 44, 281-465.

SchneIrla, T. C. (1971). Army Ants, A Study in Social Organization. San Francisco: W. H. Freeman \& Co.

Schweitzer, F., LAO, K. \& FAMiLy, F. (1995) Active random walkers simulate trunk trail formation by ants. Preprint.

Watmough, J. \& Edelstein-Keshet, L. (1995). A one dimensional model of trail propagation by army ants. J. math. Biol. 33: $459-476$. 